\title{
State of Emergency Medicine in Switzerland: a national profile of emergency departments in 2006
}

\author{
Bienvenido Sanchez ${ }^{1}$, Alexandre H Hirzel ${ }^{2}$, Roland Bingisser ${ }^{3}$, Annette Ciurea ${ }^{4}$, Aris Exadaktylos ${ }^{5}$, Beat Lehmann ${ }^{5}$, \\ Hans Matter ${ }^{6}$, Kaspar Meier ${ }^{7}$, Joseph Osterwalder ${ }^{8}$, Robert Sieber ${ }^{8}$, Bertrand Yersin ${ }^{1}$, Carlos A Camargo $\mathrm{Jr}^{9}$ and \\ Olivier Hugli ${ }^{*}$
}

\begin{abstract}
Background: Emergency departments (EDs) are an essential component of any developed health care system. There is, however, no national description of EDs in Switzerland. Our objective was to establish the number and location of EDs, patient visits and flow, medical staff and organization, and capabilities in 2006, as a benchmark before emergency medicine became a subspecialty in Switzerland.
\end{abstract}

Methods: In 2007, we started to create an inventory of all hospital-based EDs with a preliminary list from the Swiss Society of Emergency and Rescue Medicine that was improved with input from ED physicians nationwide. EDs were eligible if they offered acute care $24 \mathrm{~h}$ per day, 7 days per week. Our goal was to have 2006 data from at least 80\% of all EDs. The survey was initiated in 2007 and the 80\% threshold reached in 2012.

Results: In 2006, Switzerland had a total of 138 hospital-based EDs. The number of ED visits was 1.475 million visits or 20 visits per 100 inhabitants. The median number of visits was 8,806 per year; 25\% of EDs admitted 5,000 patients or less, 31\% 5,001-10,000 patients, 26\% 10,001-20,000 patients, and 17\% >20,000 patients per year. Crowding was reported by $84 \%$ of EDs with $>20,000$ visits/year. Residents with limited experience provided care for $77 \%$ of visits. Imaging was not immediately available for all patients: standard X-ray within 15 min (70\%), non-contrast head CT scan within 15 min (38\%), and focused sonography for trauma (70\%); 67\% of EDs had an intensive care unit within the hospital, and $87 \%$ had an operating room always available.

Conclusions: Swiss EDs were significant providers of health care in 2006. Crowding, physicians with limited experience, and the heterogeneity of emergency care capabilities were likely threats to the ubiquitous and consistent delivery of quality emergency care, particularly for time-sensitive conditions. Our survey establishes a benchmark to better understand future improvements in Swiss emergency care.

Keywords: Emergency medicine; Emergency medical services; Emergency hospital service; Switzerland

\section{Background}

Switzerland (Figure 1) has joined the growing number of European countries that have either already recognized or are in the process of recognizing emergency medicine as a specialty [1]. Up until now, there has not been a standard for emergency medicine (EM) specialty training programs or a central organization of accreditation that guarantees

\footnotetext{
*Correspondence: olivier.hugli@chuv.ch

${ }^{1}$ Emergency Department, Lausanne University Hospital, Lausanne, Switzerland

Full list of author information is available at the end of the article
}

the quality of EM care and procedures in Switzerland. Moreover, health care politics and the organization of emergency care are governed in a decentralized manner by each of the 26 states, as Switzerland is a federation [2]. Each state delegates emergency care to the emergency departments (ED) of public or private hospitals for which there are currently only non-binding recommendations as to the quality of the organization, training of health care personnel, as well as minimum technical requirements published in 2005 by the Swiss Society of Emergency and Rescue Medicine (SSERM) [3,4]. State of International 


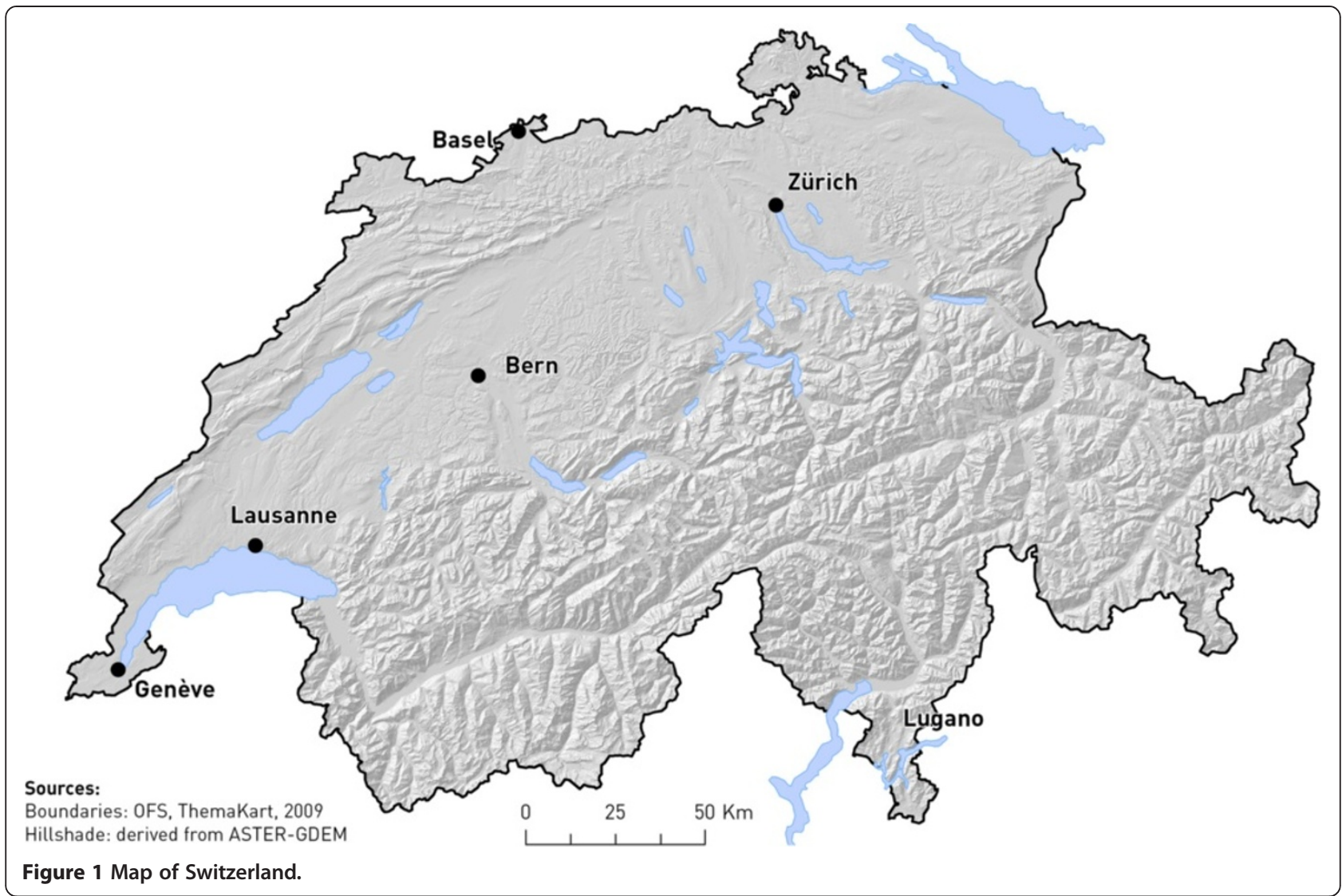

Emergency Medicine Although emergency medicine is not yet a board-certified specialty in Switzerland [5], it became a subspecialty in 2009 when the Swiss Medical Association required 18 months of additional training to receive a certificate of emergency medicine, above the Swiss Medical Association certificate requirements for general internal medicine, surgery, orthopedic surgery, cardiology, intensive care medicine, or anesthesiology [6]. The training focuses on medical and surgical emergencies of adults only [7]. The first physicians to have undergone this specialized training in emergency medicine were certified in 2011.

Whereas Switzerland, populated with 8 million inhabitants and with four national languages, has a system of public health surpassed by only the USA in per capita costs [8], to this day it does not possess a description of its EDs, an essential component of any health care system. Our objective was to establish the structural characteristics and location, the number of patient visits and flow, and the medical organization and personnel of Swiss EDs in 2006, as a benchmark before emergency medicine became a subspecialty in Switzerland.

\section{Methods}

We defined an ED as any emergency unit linked to a hospital offering acute care $24 \mathrm{~h}$ per day, 7 days per week.
EDs dedicated solely to ophthalmology or psychiatry were excluded on account of their very specialized structures and technical capabilities. In 2007, we started to create an inventory of all hospital-based EDs with a preliminary list from the SSERM. During 2008 to 2010, while conducting this unprecedented national survey, we improved on this list with input from ED physicians nationwide. For the purpose of our survey, Switzerland was divided into five zones based on linguistics or geography; one or two of the co-authors per zone sent a questionnaire to the head physician of each emergency center, or in the absence of such, to the acting head or to the hospital administration. The contents of the questionnaire were drawn up using structural and organizational recommendations for Swiss hospital EDs as far as the medical and technical aspects were concerned [4]. Other aspects of the questionnaire were drawn from the National ED Inventories (NEDI) project $[9,10]$. A teaching hospital is defined as a hospital accredited by the Swiss Medical Association for clinical education and training. "Triage to service" refers to the process of patients arriving at the ED being directed to emergency care from non-EM specialists, for example, the medical vs. surgical team. Crowding was defined as more patients than available triage rooms or beds at 6 p.m. on a typical day, while boarding was defined as a wait in the ED $>2 \mathrm{~h}$ before transfer to the hospital floors by 6 p.m. on 
a typical day [11]. The list of medical specialties was based on the NEDI list (e.g., general surgeon, anesthesiologist, orthopedic surgeon, obstetrician-gynecologist, cardiologist, pediatrician, psychiatrist, plastic surgeon neurologist, and neurosurgeon), with the addition of two specialties (i.e., internist, and radiologist) of particular importance to EM in Switzerland.

When an ED returned a partially filled questionnaire and we could not elicit another response from the site, either the national report on Swiss hospitals [12], hospital website, or annual reports from individual hospitals were queried for missing data.

For each postal code with a hospital-based ED, we applied one of the 14 Swiss spatial mobility region (SSMR) codes defined in 2000, which were based on the 2000 Swiss census [13]. Briefly, the SSMR codes were developed to measure urbanization and rural character at a regional level, and classifies each of the 106 regions of Switzerland into 14 categories based on complex sets of criteria that include the number of residents, the percentage of commuters, the relative percentage of housing and offices, the proportion of the population engaged in the primary or secondary activity sectors of the economy, as well as the number of overnight tourist stays per resident. The 14 categories are grouped into four levels: (1) metropolitan area, (2) large non-metropolitan urban area with 40,000 to 120,000 residents, (3) small non-metropolitan urban area with $<40,000$ residents, and (4) rural area [13].

We collected the data describing Swiss EDs and their activities for the period from 1 January 2006 to 31 December 2006. Our goal was to obtain a response rate of $80 \%$ of Swiss EDs, a requirement to be part of the NEDI project [10]. Our study was initiated in 2007 and the $80 \%$ threshold reached in 2012.

\section{Statistical analysis}

Continuous variables are presented using the average and standard deviation (SD), while data with a non-normal distribution are presented using the median and interquartile range (IQR). Categorical variables are presented as percentages. The results were stratified and analyzed according to the number of visits per year. Inter-group comparisons were done using ANOVA for continuous variables with a normal distribution and the KruskalWallis test for data with a non-normal distribution; categorical data was analyzed with the chi-square test or Fisher's exact test. Statistical analyses were done using Stata 12.0 (StataCorp, College Station, TX, USA). A twosided $P<0.05$ was considered statistically significant.

\section{Results}

In 2006, Switzerland had a total of 124 hospital-based EDs, or 78 EDs per 10,000 square miles, or 17 EDs per $1,000,000$ inhabitants. The total number of EDs that we surveyed was greater than the number of hospitals because some hospitals had separate and autonomous EDs for pediatrics $(n=11)$ or gynecology $(n=3)$ within the same hospital. Therefore, the total ED census was 138; 99 EDs (72\%) were located in the German-speaking region of Switzerland, 30 (22\%) in the French-speaking region, and $9(7 \%)$ in the Italian-speaking region (Figure 2). Of the 138 EDs, 122 (88\%) returned the survey, with 108 (78\%) completely filled and $14(10 \%)$ partially filled, encompassing 111 (90\%) of the 124 hospitals (Figure 2). By language region, the response rates were $84 \%$ German, 97\% French, and $100 \%$ Italian $(P=0.08)$.

\section{Number and location of the EDs}

The vast majority (95\%) of EDs were based in teaching hospitals; $9 \%$ were in university hospitals, and $87 \%$ were at public institutions. The EDs treated both adults and children in $45 \%$ of cases, uniquely adults in $46 \%$, uniquely children in $7 \%$, and only gynecology patients in $2 \%$. Most (80\%) of all EDs also served as a walk-in clinic, providing episodic care to patients without appointment, and for non-urgent conditions. Nearly 2/3 of EDs were located in a metropolitan area or a non-metropolitan city with $\geq 40,000$ residents (Table 1). The EDs were further stratified according to the average number of annual visits: 1 to 5,000 in 31 (25\%) of EDs, 5,001 to 10,000 in $38(31 \%)$, 10,001 to 20,000 in 32 (26\%), and $>20,000$ visits in 21 (17\%) of EDs (Table 1 and Figure 3). Almost all EDs with > 10,000 annual visits were located in an urban setting (Figure 4).

\section{Patient visits and flow}

In 2006, 122 EDs handled 1.475 million visits (Table 1), or 20 visits per 100 inhabitants. The median number of annual visits for each ED was 8,806 (5,196 - 16,180), with a range of 581 to 60,000 . Half (50\%) of EDs admitted $<8,760$ patients per year, or less than one patient per hour; of those, $34 \%$ were located in a metropolitan area, $20 \%$ in a large non-metropolitan urban area, $13 \%$ in a small non-metropolitan urban area, and 33\% in a rural area $(P=0.001)$.

Overall, 39\% of visits were for trauma or surgery, 32\% for internal medicine, $15 \%$ for pediatrics, and $14 \%$ for other medical specialties. Transportation to the ED was estimated to be by ambulance in 15\% (IQR 5-25). Crowding was reported at $48 \%$ of all EDs, but reached $84 \%$ among the larger centers. The ED visit capacity was judged to be insufficient for the demand at $41 \%$ of EDs, and once again the insufficiency was more marked at the larger establishments $(P=0.02)$.

After assessment in the ED, 27\% (IQR 17-33) of patients were hospitalized in the same hospital, and $2 \%$ (IQR 1-5) transferred to a different hospital; 66\% of EDs had established protocols to transfer patients to tertiary 


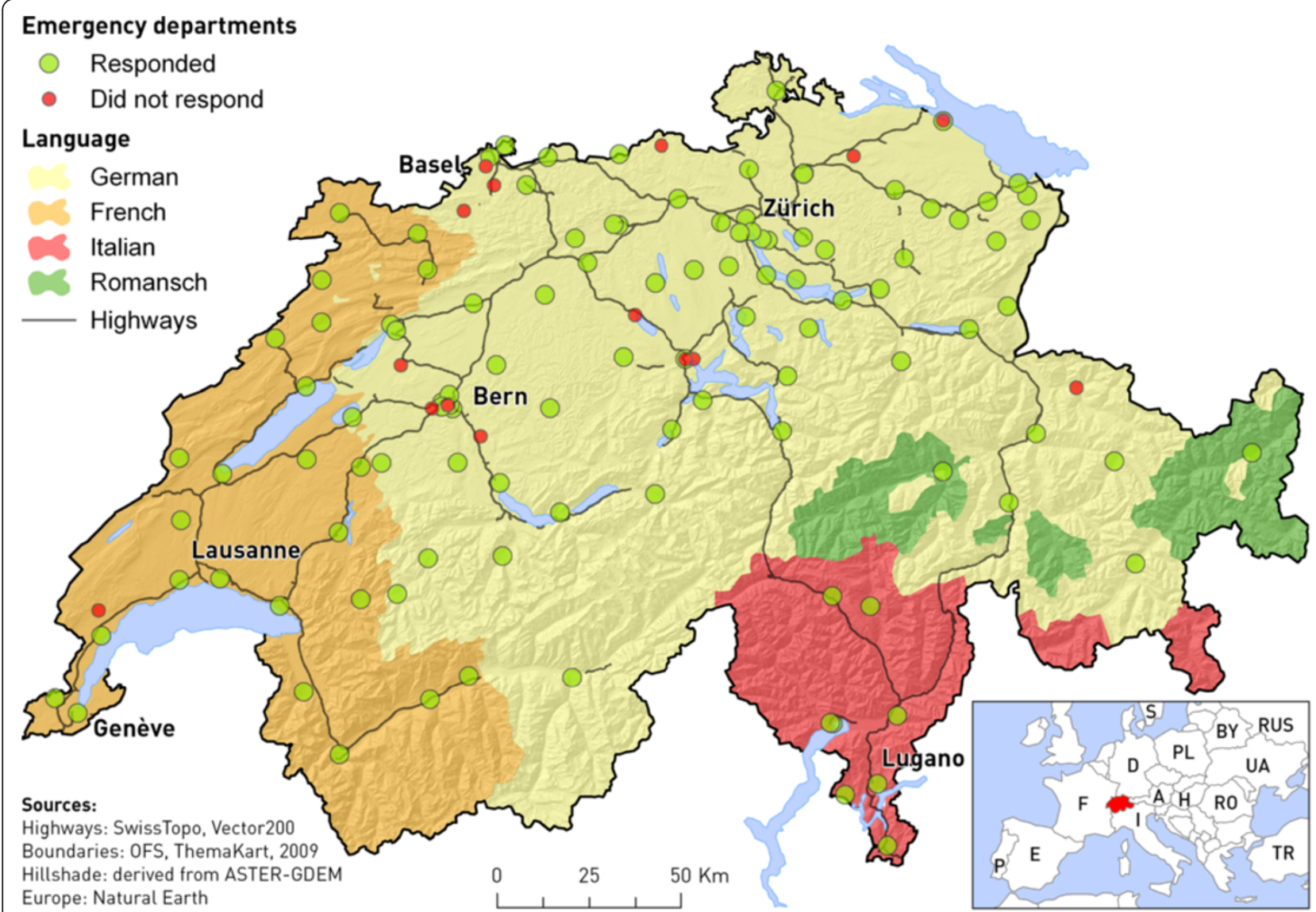

Figure 2 Emergency department locations in Switzerland.

care hospitals for specialized treatments. Boarding was reported by almost half of all EDs, and by $2 / 3$ of the largest EDs $(P<0.001)$. Two-thirds $(65 \%)$ of EDs were located in a hospital with an intensive care unit (ICU), but this percentage ranged from $23 \%$ in the smallest EDs to $94 \%$ in the largest. The capacity for admission to an acute care bed was deemed insufficient by $29 \%$ of all EDs and was 53\% among the largest EDs; the opposite was true at smaller hospitals, where overcapacity was more prevalent $(P<0.001)$. With regard to capacity in the ICU, this was deemed insufficient in $31 \%$ of EDs.

\section{Medical staff and organization}

The majority of EDs (90\%) had triage to service. Initial patient evaluation was done by trainees (residents) in $77 \%$ of cases and attending physicians in $6 \%$, while the remainder was handled by both (Table 2 ). These respective percentages varied by the size of the ED $(P=0.009)$. More than half $(58 \%)$ of all EDs had no requirement for prior postgraduate training for the residents in order to work there; one-third (35\%) of EDs required at least 6 months and 7\% required at least 24 months. The duration of ED rotation was very short for the most part: $\leq 3$ months for $51 \%$ of all
EDs with very few exceeding 6 months. The respondents from the smallest EDs favored a rotation $>6$ months, those from medium-sized EDs favored $<3$ months, and those from the largest EDs favored a 4 to 6 -month rotation $(P<$ 0.001 ). Physicians trained in advanced cardiac life support (ACLS) were present in $89 \%$ of EDs, in advanced trauma life support (ATLS) in 82\%, and in pediatric life support (PALS) in 56\%, but these individuals were not always available within $10 \mathrm{~min}$ (Table 2). Only the percentage of PALS-trained physicians was significantly higher in the larger EDs $(P=0.01)$. EDs staffed with physicians providing all three types of advanced life support were also more common in larger EDs $(P=0.002)$.

The daily availability of specialists varied greatly across specialty type, as well as across hospital size (Table 3). Smaller EDs had limited access to specialists in radiology, pediatrics, psychiatry, plastic surgery neurology, and neurosurgery specialists, with very rare access to the latter five $(14 \%, 9 \%, 8 \%, 8 \%$, and $12 \%)$.

\section{Capabilities}

With respect to available technical resources, a resuscitation room was on hand in $77 \%$ of EDs, from $56 \%$ in small 
EDs to $95 \%$ of the larger centers $(P=0.001)$. The majority of EDs had immediate access to vital laboratory tests, blood gas measurements, and EKGs (Table 4); immediate blood transfusion was available in 53\% of EDs and other blood products only in $28 \%$, both of them being similar across ED size.

All EDs had access to conventional medical imaging, but access to CT or ultrasound was less common in small EDs $(P<0.001$ and 0.016 , respectively). Only $19 \%$ of all had a CT scanner within the ED. Access to a non-contrast head CT or $\mathrm{x}$-ray in $<15$ min was less common in smaller EDs $(P=0.02$ and 0.01 , respectively). Immediate focused assessment with sonography for trauma (FAST) was available in $61 \%$ of EDs and, when available, was performed by ED physicians in $58 \%$ of cases. An operating room, dialysis, catheterization laboratory, and a stroke unit available 24/7 were more commonly found in larger EDs (all $P \leq 0.01$ ).

\section{Discussion}

Emergency care is uniquely defined by the urgency and location of treatment and transcends the usual boundaries of most organizations and medical specialties [14]. Universal provision of quality emergency care, particularly for time-sensitive conditions (such as fibrinolytic therapy for acute ischemic stroke, revascularization in acute myocardial infarction, or management of severe injury or trauma), is an essential component of any highly developed health care system, such as the Swiss health care system. The delivery of quality emergency care therefore requires physicians with broad expertise and substantial resources. Our national survey, using data from 2006, is the first to offer a global picture of EDs in Switzerland.

We found similarities as well as differences between Swiss EDs and those of other countries. First of all, the 122 Swiss EDs handled approximately 1.475 million visits or 20 visits per 100 inhabitants in 2006, a figure that underestimates the true number since only $88 \%$ of EDs responded to our survey. However, based on hospital characteristics associated with the number of ED visits, the 16 non-responding hospitals accounted for approximately 133,000 visits. The total annual number of ED visits was close to 1.5 million, therefore 1.6 times higher

Table 1 Number, location of Swiss emergency departments (EDs), and patient flow

\begin{tabular}{|c|c|c|c|c|c|c|}
\hline \multirow[t]{2}{*}{ Characteristics } & \multirow[b]{2}{*}{ All } & \multicolumn{5}{|c|}{ Number of visits per year } \\
\hline & & $1-5,000$ & $5,001-10000$ & $10,001-20,000$ & $>20,000$ & $P$ value \\
\hline Number of EDs, $n[\%]$ & 122 & $31[25]$ & $38[31]$ & $32[26]$ & $21[17]$ & \\
\hline \multicolumn{7}{|l|}{ Urban: - rural status } \\
\hline Metropolitan area [\%] & 46 & 16 & 47 & 50 & 67 & \\
\hline Large non-metropolitan urban area [\%] & 20 & 23 & 16 & 25 & 24 & $<0.001$ \\
\hline Small non-metropolitan urban area [\%] & 15 & 13 & 24 & 19 & 5 & \\
\hline Rural area [\%] & 19 & 48 & 13 & 6 & 5 & \\
\hline Total number of visits [thousands] & 1,436 & 89.6 & 283.4 & 470.4 & 631.8 & \\
\hline Median number of visits & 8,806 & 2,595 & 7,028 & 15,134 & 26,317 & \\
\hline$[\mathrm{QQR}]$ & {$[5,196-16,180]$} & {$[1,742-3,909]$} & {$[6,086-8,607]$} & {$[12,063-17,000]$} & {$[24,621-32,000]$} & \\
\hline Crowding [\%] & 48 & 17 & 32 & 69 & 84 & $<0.001$ \\
\hline \multicolumn{7}{|l|}{ ED capacity estimation : } \\
\hline Insufficient [\%] & 41 & 20 & 32 & 62 & 53 & \\
\hline Adequate [\%] & 51 & 64 & 59 & 34 & 47 & 0.021 \\
\hline Underutilized [\%] & 8 & 16 & 9 & 3 & 0 & \\
\hline Boarding [\%] & 48 & 16 & 41 & 66 & 74 & $<0.001$ \\
\hline \multicolumn{7}{|l|}{ Hospital capacity estimation: } \\
\hline Insufficient[\%] & 29 & 0 & 21 & 48 & 53 & \\
\hline Adequate [\%] & 64 & 92 & 68 & 52 & 42 & $<0.001$ \\
\hline Underutilized [\%] & 7 & 8 & 12 & 0 & 5 & \\
\hline ICU within hospital & 65 & 23 & 59 & 91 & 95 & $<0.001$ \\
\hline \multicolumn{7}{|l|}{ ICU capacity estimation: } \\
\hline Insufficient [\%] & 31 & 20 & 32 & 26 & 39 & \\
\hline Adequate [\%] & 61 & 60 & 59 & 70 & 50 & 0.69 \\
\hline Underutilized [\%] & 8 & 20 & 9 & 4 & 11 & \\
\hline
\end{tabular}

Abbreviations: IQR: interquartile range, ICU: intensive care unit. 


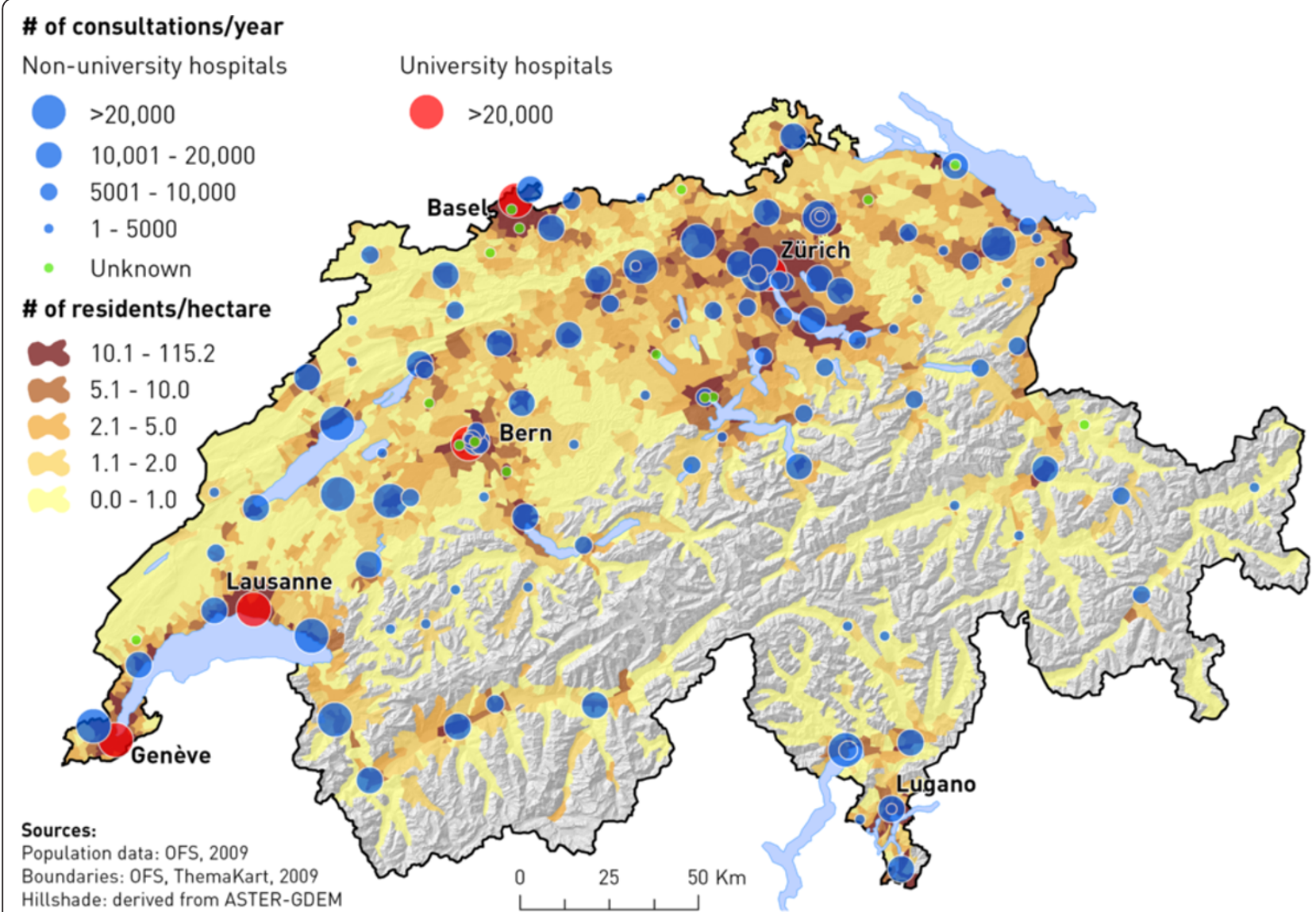

Figure 3 Emergency department visits and population density.

than the only other national estimate available $(820,000$ visits) [15], which was based on a regional sample collected in 2006. Thus, EDs are even more significant contributors to health services in Switzerland than is officially recognized. With respect to the number of inhabitants, the number of ED visits is less than, but close to that of other European countries, such as France and the UK, which each have about 24 visits per 100 inhabitants $[16,17]$. It is, however, considerably less than the 41 visits per 100 inhabitants in the USA [18], and this despite a higher density of EDs per square mile: 2.9 times higher than in France and 5.6 times higher than in the USA [19]. This discrepancy between ED visits and ED density illustrates that the quote "if you build it they will come" [20] does not always apply, as other factors such a higher physician density mitigate this correlation [21]; the Swiss population has rapid access not just to medical care at an ED within close proximity, but also to a density of office-based physicians that is 1.6 times higher than in the USA [22].

Among Swiss EDs, the activities at the different EDs varied greatly, from underutilized to crowding. Almost $60 \%$ of EDs had $\leq 10,000$ annual visits, twice the US proportion
[19]. But even more significant is the fact that half of Swiss EDs saw less than one patient per hour. These low-volume EDs typically do not have broad medico-technical resources, specialized staff, or direct access to acute-care specialists. Critical equipment such as a resuscitation or operating room and ICU were available in two-thirds or less of EDs admitting up to 10,000 patients per year. Imaging capabilities such as CT scans were also less common in low-volume EDs, and when present, took longer to obtain. Furthermore, permanent access to radiologists, pediatricians, psychiatrist, neurologists, and neurosurgeons was almost non-existent; the ability of low-volume EDs to manage time-sensitive conditions was therefore limited. This issue is circumvented in part through regionalization [23]. Although each of the independent 26 Swiss cantons has developed its own comprehensive health care system, bior multilateral arrangements between neighboring cantons have been implemented to maximize the use of highly specialized care [2], a fact illustrated by existing transfer protocols reported by two-thirds of Swiss EDs, a proportion slightly higher than in the UK [24]. However, transfer of patients to larger facilities may not be an option at any time of the year for EDs located in mountainous areas. 


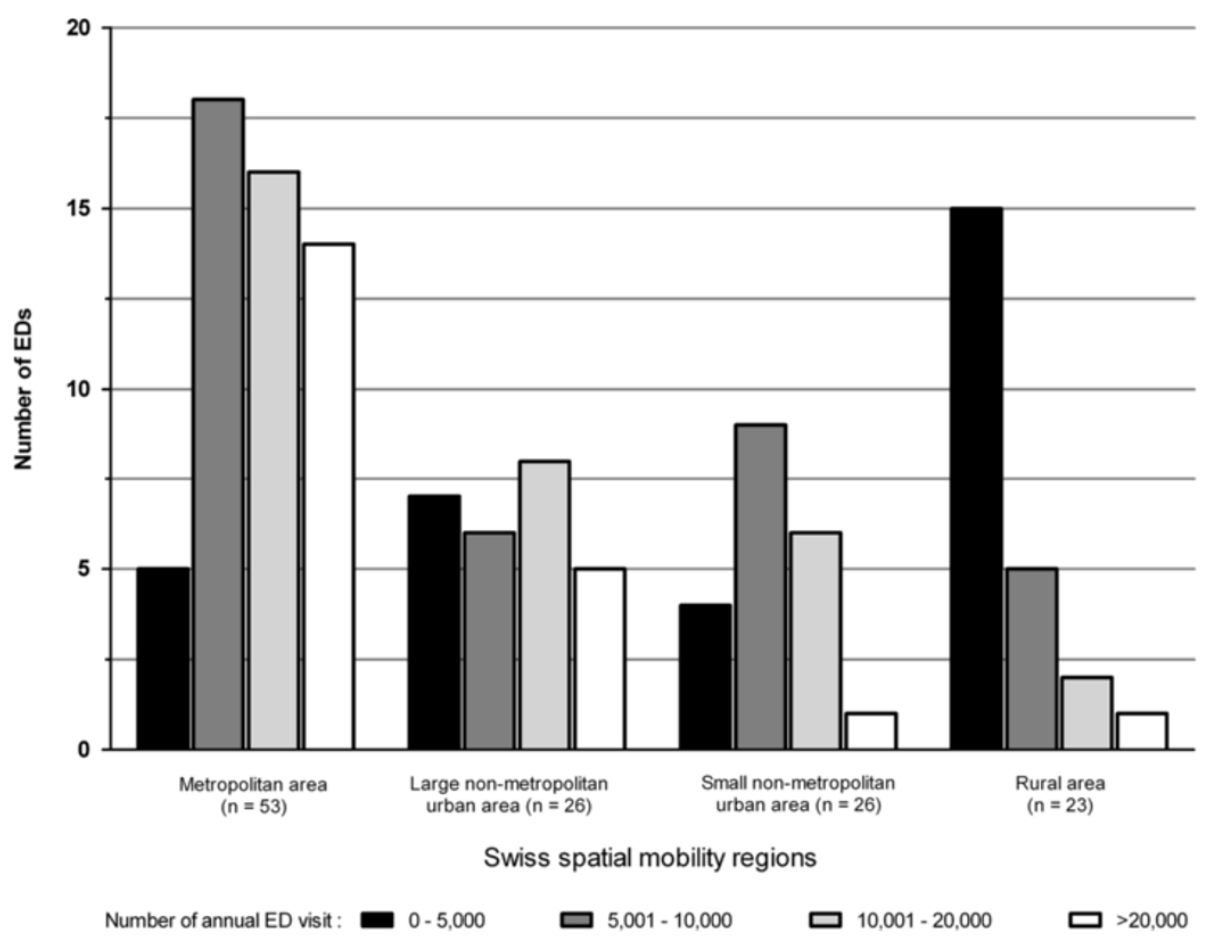

Figure 4 Number of Swiss EDs by urban-rural and annual volumes in 2006.

Table 2 Medical staff and organization

\begin{tabular}{|c|c|c|c|c|c|c|}
\hline \multirow[t]{2}{*}{ Characteristics } & \multirow[b]{2}{*}{ All } & \multicolumn{5}{|c|}{ Number of visits per year } \\
\hline & & $1-5,000$ & $5,001-10,000$ & $10,001-20,000$ & $>20,000$ & $P$ value \\
\hline \multicolumn{7}{|l|}{ Initial patient care handled by: } \\
\hline Residents only [\%] & 77 & 68 & 66 & 97 & 79 & \\
\hline Attending physicians only [\%] & 6 & 16 & 3 & 0 & 5 & 0.009 \\
\hline Both [\%] & 18 & 16 & 31 & 3 & 16 & \\
\hline \multicolumn{7}{|c|}{ Required post-graduate training for residents before ED rotation: } \\
\hline None [\%] & 58 & 62 & 70 & 57 & 33 & \\
\hline$\geq 6$ months [\%] & 35 & 38 & 18 & 39 & 56 & 0.051 \\
\hline$\geq 24$ months [\%] & 7 & 0 & 12 & 4 & 11 & \\
\hline \multicolumn{7}{|l|}{ Duration of ED rotation } \\
\hline$\leq 3$ months [\%] & 51 & 22 & 65 & 69 & 22 & \\
\hline $4-6$ months [\%] & 31 & 28 & 18 & 24 & 72 & $<0.001$ \\
\hline$>6$ months [\%] & 18 & 50 & 18 & 7 & 6 & \\
\hline \multicolumn{7}{|c|}{ Physician(s) certified in advanced life support: } \\
\hline ACLS [\%] & 89 & 91 & 85 & 93 & 88 & 0.83 \\
\hline Available <10 min [\%] & 82 & 87 & 72 & 89 & 78 & 0.59 \\
\hline ATLS [\%] & 82 & 76 & 71 & 93 & 89 & 0.14 \\
\hline Available <10 min [\%] & 71 & 64 & 52 & 89 & 83 & 0.61 \\
\hline PALS [\%] & 56 & 40 & 52 & 52 & 89 & 0.011 \\
\hline Available <10 min [\%] & 40 & 29 & 38 & 32 & 80 & 0.53 \\
\hline All 3 certifications [\%] & 46 & 23 & 38 & 52 & 82 & 0.002 \\
\hline
\end{tabular}

Abbreviations: ACLS: advanced cardiac life support, ATLS: advanced trauma life support, PALS: pediatric advanced life support. 
Table 3 Daily availability of specialized physicians

\begin{tabular}{|c|c|c|c|c|c|c|}
\hline \multirow[t]{2}{*}{ Characteristics } & \multirow[b]{2}{*}{ All } & \multicolumn{5}{|c|}{ Number of visits per year } \\
\hline & & $1-5,000$ & $5,001-10,000$ & $10,001-20,000$ & $>20,000$ & $P$ value \\
\hline General surgeon [\%] & 97 & 95 & 97 & 100 & 95 & 0.69 \\
\hline Anesthesiologist [\%] & 94 & 91 & 97 & 93 & 95 & 0.80 \\
\hline Orthopedic surgeon [\%] & 85 & 75 & 87 & 89 & 90 & 0.55 \\
\hline Obstetrician-gynecologist [\%] & 89 & 83 & 92 & 92 & 89 & 0.87 \\
\hline Cardiologist [\%] & 58 & 44 & 58 & 54 & 81 & 0.08 \\
\hline Pediatrician [\%] ${ }^{* *}$ & 56 & 14 & 68 & 53 & 90 & 0.001 \\
\hline Psychiatrist [\%] & 45 & 9 & 47 & 54 & 74 & $<0.001$ \\
\hline Plastic surgeon [\%] & 32 & 8 & 33 & 34 & 58 & $<0.01$ \\
\hline Neurologist [\%] & 29 & 8 & 26 & 25 & 68 & $<0.001$ \\
\hline Neurosurgeon [\%] & 23 & 12 & 23 & 18 & 47 & 0.052 \\
\hline Internist [\%]* & 97 & 95 & 100 & 92 & 100 & 0.40 \\
\hline Radiologist [\%] & 86 & 65 & 87 & 96 & 89 & 0.03 \\
\hline
\end{tabular}

*Pediatric-only hospitals excluded $(n=7)$.

**Adult-only hospitals excluded $(n=45)$.

In addition, regionalization often results in centralization, or a one-way transfer from smaller to larger EDs [25], and may exacerbate the crowding of high-volume EDs and their associated hospitals. Crowding threatens the quality and effectiveness of patient care across a variety of medical conditions [26] and is reported by most Swiss EDs. Crowding is often conceptualized as the result of increased demands of ED services (input), suboptimal care processes that prolong the ED stay (throughput), and finally inefficient management or placement of ED patients (output) [27]. Limited data suggest that the number of ED visits has increased significantly over time [28]. Physicians with limited experience in EM who staff Swiss and other European EDs contribute to a slower patient throughput [29]. Finally, an insufficient number of acute inpatient beds, a consequence of the significant reduction

Table 4 Capabilities

\begin{tabular}{|c|c|c|c|c|c|c|}
\hline \multirow[t]{2}{*}{ Characteristics } & \multirow[b]{2}{*}{ All } & \multicolumn{5}{|c|}{ Number of visits per year } \\
\hline & & $1-5,000$ & $5,001-10,000$ & $10,001-20,000$ & $>20,000$ & $P$ value \\
\hline Resuscitation room [\%] & 77 & 56 & 67 & 93 & 95 & 0.001 \\
\hline \multicolumn{7}{|l|}{ Laboratory tests available: } \\
\hline Vital laboratory results <30 min [\%] & 88 & 92 & 89 & 90 & 79 & 0.63 \\
\hline Blood gases < 15 min [\%] & 98 & 100 & 97 & 100 & 95 & 0.55 \\
\hline Electrocardiogram <15 min [\%] & 99 & 100 & 97 & 100 & 100 & 1.0 \\
\hline \multicolumn{7}{|l|}{ Blood products } \\
\hline Blood transfusion <15 min [\%] & 53 & 44 & 43 & 66 & 63 & 0.19 \\
\hline Other blood products <15 min [\%] & 28 & 20 & 29 & 38 & 21 & 0.49 \\
\hline \multicolumn{7}{|l|}{ Medical imaging: } \\
\hline X-ray (<15 min), [\%] & $100(70)$ & $100(60)$ & $100(57)$ & $100(90)$ & $100(79)$ & $-(0.014)$ \\
\hline$C T$ (non-contrast head CT <15 min), [\%] & $87(38)$ & $60(16)$ & $92(34)$ & $100(55)$ & $95(47)$ & $<0.001(0.019)$ \\
\hline Ultrasound (<15 min), [\%] & $97(56)$ & $88(72)$ & $100(51)$ & $100(59)$ & $100(42)$ & $0.016(0.22)$ \\
\hline FAST immediately available (performed by ED physician), [\%] & $61(58)$ & $48(70)$ & $48(60)$ & $76(62)$ & $72(36)$ & $0.07(0.5)$ \\
\hline \multicolumn{7}{|l|}{ 24/7 availability of: } \\
\hline Operating room [\%] & 87 & 64 & 91 & 97 & 95 & $<0.01$ \\
\hline Dialysis [\%] & 41 & 8 & 29 & 62 & 74 & $<0.001$ \\
\hline Catheterization laboratory [\%] & 21 & 8 & 20 & 14 & 48 & 0.01 \\
\hline Stroke unit [\%] & 19 & 4 & 14 & 21 & 45 & 0.007 \\
\hline
\end{tabular}

Abbreviations: FAST focused assessment with sonography for trauma. 
of acute care hospital beds since 1990 in Switzerland [22], adversely affects output.

The lack of clinical experience in EM is a concern not just with regard to crowding but also to the quality of care delivered to the most critically ill ED patients. Junior residents may lack the necessary knowledge of EM because of the current deficient undergraduate teaching of acute care medicine in European medical schools [30]. Recent graduates of Swiss medical schools have suboptimal competencies to work up or treat common [31] or critical emergency situations [32]. Their intermittent or shortduration work in the ED during resident training may be too little exposure to EM to solidify their knowledge and skills in this field; this may be further aggravated by the lack of or limited supervision during night and weekend shifts [5]. Recent developments in the status of EM as a subspecialty provide an opportunity for certified Swiss ED physicians in the ED to participate in the undergraduate and postgraduate teaching of EM in Swiss medical schools, both in the lecture hall and at the bedside [33]. We anticipate that the greater involvement of ED physicians in teaching and in the direct care of patients will improve residents' skills and patient outcomes, an improvement that we hope to demonstrate in future national surveys.

The vast majority of EDs used triage to service, which reflects the absence of EM-trained specialists in this country. This proportion is higher than in other European countries such as Denmark (54\%) [34], but closer to others like Slovenia (100\%) [35]. A substantial body of literature highlights the benefits of EM-trained physicians for the care of critically ill patients [36,37]. However, a large proportion of Swiss EDs have such a low volume of patients that a dedicated team of ED physicians is not clinically or financially feasible; this problem has also been reported in other countries [38]. Closure of smaller EDs may be an option given the relatively small size of Switzerland, especially for EDs in densely populated areas; however, it may deprive remote areas of their access to emergency care. Previous attempts to close smaller EDs have in fact been met with strong opposition from the voters, and politicians have been forced to withdraw all such proposals in the past [2]. As suggested, one alternative to dedicated ED physicians is to integrate EM core curricula into primary care programs such as internal or family medicine [39]. Another approach is to provide high-quality emergency care through telemedicine, a field not yet developed in Switzerland. Observational studies suggest a beneficial impact of telemedicine for pediatric $[40,41]$, neurosurgery [42], stroke [43], and both major and minor trauma cases $[44,45]$, all of which require medical specialists that are rarely available in smaller Swiss EDs.

Finally, our study highlights the heterogeneity of emergency care capabilities provided by Swiss EDs. Although high-volume EDs provided more comprehensive services in general, there were many exceptions. There is no explicit ED categorization to date, although classification criteria have been published in 2005 [4]. The establishment of a map detailing the available competencies and resources of all Swiss EDs is essential to guide individual patients or Emergency Medical Systems to the appropriate ED; categorization may also provide decision-makers with the information required to plan emergency care at the regional and national level $[46,47]$. Providing such a list is in fact a requirement for EDs who want to be certified by the SSERM as training programs for EM.

Our study provides the largest, most inclusive perspective on Swiss EDs to date with participation of $88 \%$ of all Swiss EDs. Our survey was conducted 6 years ago, a delay explained by the difficulty in creating a complete national list and reaching a response rate of $80 \%$; however, our goal is to provide data that will serve as a benchmark to assess future progress and allow for international comparisons. Another limitation is that data were collected over a 6-year period, and we therefore cannot exclude a recall bias. However, approximately $75 \%$ of questionnaires were returned in 2007 and 2008, minimizing the potential magnitude of this issue. Our survey did not include any emergency care performance measures, such as those developed in the Physician Quality Reporting Initiative developed by the Centers for Medicare \& Medicaid Services (CMS) [48]. Therefore, the quality of care provided in Swiss EDs cannot be inferred from our data, but constitutes a field for future research projects. In one study assessing patients' experience in a limited number of Swiss EDs, patients reported satisfaction with their care management in the ED [49]. Finally, these data were self-reported, and we could not verify their complete accuracy.

\section{Conclusion}

In 2006, Switzerland had a high density of hospital-based EDs with a significantly larger number of ED visits than previously estimated. Our national survey demonstrates that EDs play a significant role in the delivery of health care for the Swiss population. It also identified crowding and the provision of emergency care by physicians with limited experience in EM as potential threats to the universal delivery of quality emergency care, particularly for time-sensitive conditions. Further studies are needed to assess the quality of care in Swiss EDs. Along those lines, our survey establishes a benchmark at the inception of major changes in Swiss emergency care and will promote a better understanding of the importance and eventual impact of planned improvements in Swiss emergency care.

\section{Abbreviations}

ACLS: Advanced cardiac life support; ATLS: Advanced trauma life support: ED: Emergency department; EM: Emergency medicine; EMNet: Emergency Medicine Network; FAST: Focused assessment with sonography for trauma; IQR: Interquartile range; NEDI: National Emergency Department Inventory; PALS: Pediatric advanced life support; SD: Standard deviation; SSERM: Swiss Society of Emergency and Rescue Medicine. 


\section{Competing interests}

The authors declare that they have no competing interests.

\section{Authors' contribution}

$\mathrm{BS}, \mathrm{BY}, \mathrm{CC}, \mathrm{KM}$, and $\mathrm{OH}$ contributed to the creation of the questionnaire; $\mathrm{RB}$, $A C, A D, B L, H M, K M, J O, R S$, and $\mathrm{OH}$ collected the data; $\mathrm{AH}$ and $\mathrm{OH}$ generated the geographical analyses; $\mathrm{OH}$ performed the statistical analyses; all authors critically revised the manuscript. All authors read and approved the final manuscript.

\section{Acknowledgements}

We thank Ulrich Burgi (Aarau), Dagmar Keller (Zürich), Olivier Rutschmann (Geneva), Markus Schwendinger (Baden), and Fabrice Dami (Lausanne) for their help in completing the survey and enrolling additional EDs.

\section{Author details}

${ }^{1}$ Emergency Department, Lausanne University Hospital, Lausanne, Switzerland. 'University of Lausanne, Lausanne, Switzerland. ${ }^{3}$ Department of Emergency Medicine, University Hospital, Basel, Switzerland. ${ }^{4}$ Emergency Department, Bulach Hospital, Bulach, Switzerland. ${ }^{5}$ Emergency Department, University Hospital Inselspital, Bern, Switzerland. 'Emergency Department, Limmattal Hospital, Schlieren, Switzerland. ${ }^{7}$ Anesthesiology Department, Regional Hospital, Ilanz, Switzerland. ${ }^{8}$ Emergency Department, Cantonal Hospital, Sankt Gallen, Switzerland. 'Department of Emergency Medicine, Massachusetts General Hospital, Harvard Medical School, Boston, MA 02114, USA.

Received: 11 September 2012 Accepted: 10 February 2013

Published: 10 July 2013

\section{References}

1. Fleischmann T, Fulde G: Emergency medicine in modern Europe. Emerg Med Australas 2007, 19:300-302.

2. Wyss K, Lorenz N: Decentralization and central and regional coordination of health services: the case of Switzerland. Int J Health Plann Manage 2000, 15:103-114.

3. Bernoulli L, Anselmi L: Programme de médecine d'urgence hospitalière SSMUS. Bull Med Suisses 2038, 2005:86.

4. Groupe de travail de la Communauté d'intérêt des directeurs médicaux des urgences: Centres d'urgence hospitaliers: recommandations structurelles et organisationnelles en matière d'assurance qualité. Bull Méd Suisses 2005, 86:1974-1984.

5. Osterwalder JJ: Emergency medicine in Switzerland. Ann Emerg Med 1998, 32:243-247.

6. Médecine d'urgence hospitalière (SSMUS): Attestation de formation complémentaire du ler juillet; 2009. http://www.fmh.ch/files/pdf7/ fa_kl_notfallmedizin_f.pdf.

7. Swiss Society of Emergency Medicine: Swiss clinical emergency medicine specific learning objectives. http://www.sgnor.ch/uploads/

tx_frptaggeddownloads/knm_Izk_2009.pdf.

8. Anderson GF, Reinhardt UE, Hussey PS: Petrosyan V: It's the prices, stupid: why the United States is so different from other countries. Health Aff (Millwood) 2003, 22:89-105.

9. Sullivan AF, Richman IB, Ahn CJ, Auerbach BS, Pallin DJ, Schafermeyer RW, Clark S, Camargo CA Jr: A profile of US emergency departments in 2001. Ann Emerg Med 2006, 48:694-701.

10. Steptoe AP, Corel B, Sullivan AF, Camargo CA Jr: Characterizing emergency departments to improve understanding of emergency care systems. Int $J$ Emerg Med 2011, 4:42.

11. Schneider SM, Gallery ME, Schafermeyer R, Zwemer FL: Emergency department crowding: a point in time. Ann Emerg Med 2003, 42:167-172.

12. Federal Office of Public Health: Swiss hospital key figures in 2006. http:// www.bag.admin.ch/shop/00102/00540/index.html?lang=fr\&download= NHzLpZig7t,Inp6I0NTU042I2Z6In1ae2IZn4Z2qZpnO2Yuq2Z6gpJCHeoR9 f2ym162dpYbUzd,Gpd6emK2Oz9aGodetmqaN19XI2ldvoaCVZ,s.

13. Schuler $M$, Dessemontet $P$, Joye $D$, Perlik $M$ : Les niveaux géographiques de la Suisse. Neuchatel: Office fédéral de la statistique; 2005.

14. Institute of Medicine (US) Committee on the Future of Emergency Care in the United States Health System: Hospital-based emergency care: at the breaking point. Washington DC: National Academies Press; 2007.
15. Guntensperger U, Pinzello-Hurlimann R, Martina B, Ciurea A, Muff B, Gutzwiller JP: Primary care emergency services utilization in Germanspeaking Switzerland: a population-based cross-sectional study. Swiss Med Wkly 2010, 140:w13111.

16. Carrasco V: L'activité des services d'urgences en 2004 - Une stabilisation du nombre de passages. In Etudes et résultats. Paris: Ministère de la santé et des sports; 2006.

17. The NHS information centre: Accident and emergency attendances in England (experimental statistics) 2007-2008. https://catalogue.ic.nhs.uk/publications/ hospital/AandE/acci-emer-atte-eng-2007-2008/acci-emer-atte-eng-20072008-rep.pdf.

18. Pitts SR, Niska RW, Xu J, Burt CW: National hospital ambulatory medical care survey: 2006 emergency department summary. Natl Health Stat Report 2008, 7:1-38.

19. Muelleman RL, Sullivan AF, Espinola JA, Ginde AA, Wadman MC, Camargo CA Jr: Distribution of emergency departments according to annual visit volume and urban-rural status: implications for access and staffing. Acad Emerg Med 2010, 17:1390-1397.

20. Taylor TB: Threats to the health care safety net. Acad Emerg Med 2001, 8:1080-1087.

21. Richman IB, Clark S, Sullivan AF, Camargo CA Jr: National study of the relation of primary care shortages to emergency department utilization. Acad Emerg Med 2007, 14:279-282.

22. Organisation for Economic Co-operation and Development: Health at a glance 2007- OECD indicators. Paris France: OECD; 2007.

23. Carr BG, Matthew Edwards J, Martinez R: Regionalized care for time-critical conditions: lessons learned from existing networks. Acad Emerg Med 2010, 17:1354-1358

24. Stevenson A, Fiddler C, Craig M, Gray A: Emergency department organisation of critical care transfers in the UK. Emerg Med J 2005, 22:795-798.

25. Rokos IC, Sanddal ND, Pancioli AM, Wolff C, Gaieski DF: Inter-hospital communications and transport: turning One-way funnels into Two-way networks. Acad Emerg Med 2010, 17:1279-1285.

26. Bernstein SL, Aronsky D, Duseja R, Epstein S, Handel D, Hwang U, McCarthy M, John McConnell K, Pines JM, Rathlev N, et al: The effect of emergency department crowding on clinically oriented outcomes. Acad Emerg Med 2009, 16:1-10.

27. Asplin BR, Magid DJ, Rhodes KV, Solberg LI, Lurie N, Camargo CA Jr: A conceptual model of emergency department crowding. Ann Emerg Med 2003, 42:173-180.

28. Santos-Eggimann B: Increasing use of the emergency department in a Swiss hospital: observational study based on measures of the severity of cases. BMJ 2002, 324:1186-1187.

29. Jayaprakash N, O'Sullivan R, Bey T, Ahmed SS, Lotfipour S: Crowding and delivery of healthcare in emergency departments: the European perspective. West J Emerg Med 2009, 10:233-239.

30. Smith CM, Perkins GD, Bullock I, Bion JF: Undergraduate training in the care of the acutely ill patient: a literature review. Intensive Care Med 2007, 33:901-907.

31. Saxer T, Duperrex O, Vermeulen B, Vu Nu V: Emergency medicine training: a prospective, comparative study of an undergraduate clinical clerkship and an army programme. Swiss Med Wkly 2009, 139:423-429.

32. Luscher F, Hunziker S, Gaillard V, Tschan F, Semmer NK, Hunziker PR, Marsch S: Proficiency in cardiopulmonary resuscitation of medical students at graduation: a simulator-based comparison with general practitioners. Swiss Med Wkly 2010, 140:57-61.

33. Bingisser R: Emergency medicine training: is undergraduate training sufficient for the Swiss health-care system? Swiss Med Wkly 2009, 139:406.

34. Wen LS, Anderson PD, Stagelund S, Sullivan AF, Camargo CA Jr: National survey of emergency departments in Denmark. Eur J Emerg Med 2013, 20:205-209.

35. Jaklic B, Wen L, Sullivan A, Camargo C Jr: A profile of emergency departments in Slovenia. ISRN Emerg Med 2012, Article ID 461274:6.

36. Hallas P: The effect of specialist treatment in emergency medicine. A survey of current experiences. Scand J Trauma Resusc Emerg Med 2006, 14:5-8.

37. Holliman CJ, Mulligan TM, Suter RE, Cameron P, Wallis L, Anderson PD, Clem $\mathrm{K}$ : The efficacy and value of emergency medicine: a supportive literature review. Int J Emerg Med 2011, 4:44.

38. Casey MM, Wholey D, Moscovice IS: Rural emergency department staffing and participation in emergency certification and training programs. J Rural Health 2008, 24:253-262. 
39. Williams JM, Ehrlich PF, Prescott JE: Emergency medical care in rural America. Ann Emerg Med 2001, 38:323-327.

40. Franken EA Jr, Berbaum KS, Brandser EA, D'Alessandro MP, Schweiger GD, Smith WL: Pediatric radiology at a rural hospital: value of teleradiology and subspecialty consultation. Am J Roentgenol 1997, 168:1349-1352.

41. Heath B, Salerno R, Hopkins A, Hertzig J, Caputo M: Pediatric critical care telemedicine in rural underserved emergency departments. Pediatr Crit Care Med 2009, 10:588-591.

42. Servadei F, Antonelli V, Mastrilli A, Cultrera F, Giuffrida M, Staffa G: Integration of image transmission into a protocol for head injury management: a preliminary report. Br J Neurosurg 2002, 16:36-42.

43. Schwamm LH, Holloway RG, Amarenco P, Audebert HJ, Bakas T, Chumbler NR, Handschu R, Jauch EC, Knight WA, Levine SR, et al: A review of the evidence for the use of telemedicine within stroke systems of care: a scientific statement from the american heart association/american stroke association. Stroke 2009, 40:2616-2634.

44. Benger JR, Noble SM, Coast J, Kendall JM: The safety and effectiveness of minor injuries telemedicine. Emerg Med J 2004, 21:438-445.

45. Ricci MA, Caputo M, Amour J, Rogers FB, Sartorelli K, Callas PW, Malone PT: Telemedicine reduces discrepancies in rural trauma care. Telemed J E Health 2003, 9:3-11.

46. National Research Council (US). Committee on Trauma, National Research Council (US). Committee on Shock: Accidental death and disability: the neglected disease of modern society. Rockville, MD: Reprinted by the U.S. Division of Emergency Health Services; 1971.

47. Mehrotra A, Sklar DP, Tayal VS, Kocher KE, Handel DA, Myles Riner R: Important historical efforts at emergency department categorization in the United States and implications for regionalization. Acad Emerg Med 2010, 17:e154-e160.

48. Centers for Medicare \& Medicaid Services: US department of health and human services medicare hospital value-based purchasing. Options paper: 2nd public listening session; 2007. https:/www.cms.gov/AcutelnpatientPPS/ downloads/HospitalVBPOptions.pdf.

49. Schwappach DL, Blaudszun A, Conen D, Ebner H, Eichler K, Hochreutener MA: 'Emerge': benchmarking of clinical performance and patients' experiences with emergency care in Switzerland. Int J Qual Health Care 2003, 15:473-485.

doi:10.1186/1865-1380-6-23

Cite this article as: Sanchez et al.: State of Emergency Medicine in Switzerland: a national profile of emergency departments in 2006. International Journal of Emergency Medicine 2013 6:23.

\section{Submit your manuscript to a SpringerOpen ${ }^{\circ}$ journal and benefit from:}

- Convenient online submission

- Rigorous peer review

- Immediate publication on acceptance

- Open access: articles freely available online

- High visibility within the field

- Retaining the copyright to your article 